\begin{tabular}{|c|c|}
\hline Title & Fast A naly sis of Rotating Machine U sing Simplified Model-Order Reduction Based on POD \\
\hline Author(s) & Sakamoto, Hiroki; Okamoto, Kazuya; Igarashi, Hajime \\
\hline Citation & $\begin{array}{l}\text { IEEE transactions on magnetics, 56(2), } 7506104 \\
\text { https://doi.org/10.1109/T MA G.2019.2947300 }\end{array}$ \\
\hline Issue Date & $2020-02$ \\
\hline Doc URL & http://hdl.handle.net/2115/76843 \\
\hline Rights & $\begin{array}{l}\text { (c) } 2020 \text { IEEE. Personal use of this material is permitted. Permission from IEEE must be obtained for all other uses, in } \\
\text { any current or future media, including reprinting/republishing this material for advertising or promotional purposes, } \\
\text { creating new collective works, for resale or redistribution to servers or lists, or reuse of any copyrighted component of } \\
\text { this work in other works. }\end{array}$ \\
\hline Tyре & article (author version) \\
\hline File Information & TMA G2947300.pdf \\
\hline
\end{tabular}

Instructions for use 


\title{
Fast Analysis of Rotating Machine Using Simplified Model Order Reduction Based on POD
}

\author{
Hiroki Sakamoto ${ }^{1,2}$, Kazuya Okamoto ${ }^{1}$, Hajime Igarashi ${ }^{1}$ \\ ${ }^{1}$ Graduate School of Information Science and Technology, Hokkaido University, Sapporo, 060-0814, Japan, \\ ${ }^{2}$ Meidensha Co., 2-1-1 Osaki, Shinagawa-ku, Tokyo, 141-8565 Japan
}

\begin{abstract}
This paper proposes a simplified model order reduction for the fast dynamic simulation of electric motors. The magnetic fields are snapshotted for different input currents at each mechanical angle to construct a data matrix. The basis vectors are then computed by the singular value decomposition applied to the data matrix. The interpolation along the mechanical angle is performed by the dynamic mode decomposition. Fast computation of the magnetic field for arbitrary input current and mechanical angle is performed through interpolation of the basis vectors in the space of input currents for the dynamic analysis of motors.
\end{abstract}

Index Terms - Rotating machine, Behavior model, Proper Orthogonal Decomposition, Dynamic Mode Decomposition, Model order reduction.

\section{INTRODUCTION}

$\mathrm{C}$ urrently, in the development and design of electric motors for electric vehicles, electromagnetic field analysis using finite element method (FEM) has widely been used. Fast dynamic simulation of a motor and control system including inverters and gears has been required for such processes. It is difficult to perform the dynamic FE analysis for this purpose due to its large computing cost. Instead of the direct use of FEM, fast simulation has been performed based on the simplified approach referred to as phase-variable model [1] or behavior model or [2]. In this approach, FEM is used in the preprocessing to compute the magnetic flux for various currents and mechanical angles. During the dynamic simulation, the magnetic flux is evaluated from the reference table or response surface. Although this approach is fast enough to simulate the dynamic behavior of the motor and control system, it is difficult to evaluate iron and copper losses and electromagnetic forces acting on the stator during the simulation because magnetic fields would have to be re-computed by FEM.

The computing cost of FEM can be reduced using the model order reduction (MOR) for the dynamic simulation of motors, for which the proper orthogonal decomposition (POD) would be effective $[3,4]$. In this method, the nonlinear reduced field equation is solved at each mechanical angle. Because the number of unknowns included in the reduced equation is small, the computing efficiency is improved in comparison with the conventional FEM. However, POD-based MOR would not work fast enough for the real-time dynamic motor simulations.

To overcome this problem, the interpolation method which does not solve the reduced equations has been proposed [5], [6]. In this method, the machine response for arbitrary input is

Manuscript received April 1, 2015; revised May 15, 2015 and June 1, 2015; accepted July 1, 2015. Date of publication July 10, 2015; date of current version July 31, 2015. (Dates will be inserted by IEEE; "published" is the date

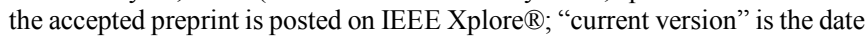
the typeset version is posted on Xplore $\left.{ }^{\circledR}\right)$. Corresponding author: $\mathrm{H}$. Sakamoto (e-mail: sakamoto_hi@eis.hokudai.ac.jp).

Color versions of one or more of the figures in this paper are available online at http://ieeexplore.ieee.org.

Digital Object Identifier (inserted by IEEE). obtained via interpolation of the basis vectors obtained by POD. The nonlinear interpolation is applied to the Grassmann manifold in [5], while the interpolation is adopted for the righthand vector of the singular value decomposition in [6].

In this work, we develop a simplified POD-based MOR for the dynamic simulation of motors. In this method, the magnetic fields are not directly stored but the basis vectors constructed by POD as well as the weighting coefficients are stored for the restoration of the magnetic field. The interpolation is done for the field in the space and time spanned by the d- and q-axis currents at each mechanical angle. The proposed method works fast because it does not solve the nonlinear equation. Dynamic simulation using the simplified POD-based MOR is discussed in [7]. The novelty of this study compared to [5-7] is the use of the dynamic mode decomposition (DMD) for the interpolation along the mechanical angle.

\section{PROPOSED METHOD}

\section{A. Simplified POD-based MOR}

The static magnetic fields are computed using FEM for different $\mathrm{d}$ and $\mathrm{q}$ axis currents, $i_{d}, i_{q}$, at mechanical angle $\theta$. The data matrix is then constructed from the resulting field as

$$
\mathrm{X}=\left[\boldsymbol{a}_{11}, \boldsymbol{a}_{21}, \cdots, \boldsymbol{a}_{s 1}, \boldsymbol{a}_{12}, \cdots, \boldsymbol{a}_{j t}, \cdots, \boldsymbol{a}_{s m}\right]
$$

where $\mathrm{X} \in \mathbb{R}^{n \times s m}, n$ is the total number of nodes for FEM and $j=1,2, \ldots, s$ and $t=1,2, \ldots, m$ represent the indices relevant to the snapshots at grid points $\left(i_{d}, i_{q}\right)$ and $\theta$, respectively. Then, we apply the singular value decomposition to $\mathrm{X}$ to obtain

$$
\mathrm{X}=\mathrm{W} \Sigma \mathrm{V}^{*}
$$

where $\mathrm{W} \in \mathbb{R}^{n \times s m}$ and $\mathrm{V} \in \mathbb{R}^{s \times s m}$ are the orthogonal matrices containing the left and right-hand eigenvectors, and $\Sigma \in$ $\mathbb{R}^{s m \times s m}$ denotes the diagonal matrix that includes the singular values $\sigma_{i}$ of $\mathrm{X}, i=1,2, \ldots, \mathrm{sm}$.

The low rank approximation with the dominant $s_{0}$ singular value is applied to (2) as

$$
\mathrm{X} \approx \widehat{\mathrm{W} C},
$$


where $s_{0} \ll s m, \widehat{\mathrm{W}} \in \mathbb{R}^{n \times s_{0}}, \mathrm{C} \in \mathbb{R}^{s_{0} \times s m}$. Accordingly, the vector potential can also be represented by the linear combination of the dominant eigenvectors as follows:

$$
a \approx \widehat{W} c
$$

where $\boldsymbol{c}$ is the coefficient vector. In the analysis of magnetostatic field based on conventional POD, the reduced FE equation for $c \in \mathbb{R}^{s_{0}}$ is solved under the assumption of (4). Since the reduced FE equation has nonlinearity due to the magnetic saturation in a motor core, its solution would still need long computing time for the real-time dynamic simulation of a motor. The proposed method does not solve the reduced FE equation but obtain the reduced variable $\boldsymbol{c}$ via interpolation in the time and space spanned by $i_{d}, i_{q}, \theta$ as will be shown in the next section.

\section{B. Interpolation for input current}

Let us consider the interpolation on the plane of $\left(i_{d}, i_{q}\right)$. The magnetic field at an arbitrary point $\left(i_{d}, i_{q}, \theta\right)$ can be computed from $\boldsymbol{a} \approx \widehat{\mathrm{W}} \boldsymbol{c}(\theta)$ where the coefficient $\boldsymbol{c}(\theta)$ is evaluated on the basis of the interpolation function used for the FE analysis with quadrature elements as

$$
\boldsymbol{c}\left(i_{d}, i_{q}, \theta\right) \approx \sum_{j=1}^{4} \boldsymbol{c}_{j}(\theta) N_{j}\left(i_{d}, i_{q}\right)
$$

The interpolation (5) is expected to work well for the fixed mechanical angle $\theta$. To consider the dependence $c$ of on $\theta$, the simplest way is to adopt the linear interpolation between sampling angles, as in [7]. This simple method would become inaccurate as the sampling interval $\Delta \theta$ becomes large. In this paper, we introduce the interpolation along the mechanical angle seeking for higher accuracy below.

\section{Interpolation for mechanical angle with DMD}

DMD performs model order reduction in time for nonlinear dynamic systems [8]. So far, DMD has been used in fluid dynamics [9] and analysis of an electric motor [10].

We consider here the interpolation along the mechanical angle $\theta$. For simplicity, we omit the index relevant to $i_{d}, i_{q}$ until notified. We assume that the change in the coefficient obeys

$$
\boldsymbol{c}(\theta+\Delta \theta)=\mathrm{A}_{d} \boldsymbol{c}(\theta)
$$

where $\mathrm{A}_{d}$ denotes the transform matrix. Moreover, we consider the continuous counterpart of (6) as

$$
\frac{d \boldsymbol{c}}{d \theta}=\mathrm{A}_{c} \boldsymbol{c}
$$

The solution to (7) can be formally represented as $\boldsymbol{c}(\theta)=$ $e^{\mathrm{A}_{c} \theta} \boldsymbol{c}_{0}$. The eigenvalue decomposition is applied to $\mathrm{A}_{c}$ to have $\mathrm{A}_{c}=\mathrm{P} \Lambda \mathrm{P}^{*}$, where $\mathrm{P}$ and $\Lambda$ are the matrix composed of the eigenvectors and diagonal matrix composed of the eigenvalues $\lambda_{i}$ of $\mathrm{P}$, respectively. The solution to (7) can be now written as $\boldsymbol{c}=\mathrm{P} e^{\Lambda_{c} \theta} \mathrm{P}^{*} \boldsymbol{c}_{0}$, where $e^{\Lambda_{c} \theta}=\operatorname{diag}\left[e^{\lambda_{1} \theta}, e^{\lambda_{2} \theta}, \ldots\right]$.

We consider here the relationship between (6) and (7). By representing $\boldsymbol{c}(\theta+\Delta \theta)$ in (6) with the solution to (7), we find

$$
\boldsymbol{c}(\theta+\Delta \theta)=e^{\mathrm{A}_{c} \Delta \theta} \boldsymbol{c}(\theta)
$$

This suggests that $\mathrm{A}_{d}=e^{\mathrm{A}_{c} \Delta \theta}$. Moreover, it can be found that

$$
\mathrm{A}_{d} \mathrm{P}=e^{\mathrm{A}_{c} \Delta \theta} \mathrm{P}=\mathrm{P} e^{\Lambda_{c} \Delta \theta}
$$

It follows from (9) that the eigenvalues of $\mathrm{A}_{d}$ are $e^{\lambda_{i} \Delta \theta}$ and the eigenvectors of $A_{d}$ and $A_{c}$ are identical. Inversely, the eigenvalues $\lambda_{i}$ of $A_{c}$ can be expressed by means of the eigenvalues $\mu_{i}$ of $\mathrm{A}_{d}$ as $\lambda_{i}=\ln \mu_{i} / \Delta \theta$.

The coefficient in (5) can be thus written as

$$
\boldsymbol{c}(\theta)=\mathrm{P} e^{\frac{\ln \Lambda_{d}}{\Delta \theta} \theta} \boldsymbol{b}
$$

where $e^{\Lambda_{d} \theta}=\operatorname{diag}\left[e^{\mu_{1} \theta}, e^{\mu_{2} \theta}, \ldots\right]$ and $\boldsymbol{b}=\mathrm{P}^{*} \boldsymbol{c}(0)$. Finally, we consider the computation of $\mu_{i}$ and $\mathrm{P}$. We introduce the matrix $C_{j, 1: m} \in \mathbb{R}^{s_{0} \times m}$ composed of the coefficient vectors $\boldsymbol{c}_{j t}$ defined by

$$
\mathrm{C}_{j, 1: m}=\left[\boldsymbol{c}_{j 1}, \boldsymbol{c}_{j 2}, \cdots, \boldsymbol{c}_{j \mathrm{~m}}\right]
$$

Now the time evolution of $C_{j}$ is assumed to obey

$$
\mathrm{C}_{j, 2: m}=\mathrm{A}_{d} \mathrm{C}_{j, 1: m-1}
$$

Moreover, for computational efficiency, $\mathrm{A}_{d}$ is projected onto the POD basis $U$ of $C_{j, 1: m-1}$ to obtain the approximation

$$
\widehat{\mathrm{A}}_{d}=\mathrm{U}^{*} \mathrm{~A}_{d} \mathrm{U}=\mathrm{U}^{*} \mathrm{C}_{j, 2: m} \mathrm{ZS}^{-1},
$$

where the matrices $\mathrm{U}, \mathrm{Z}$ and $\mathrm{S}$ are obtained from the singular value decomposition; $\mathrm{C}_{j, 1: m-1}=\mathrm{USZ}^{*}$. Note that $\mathrm{U}, \mathrm{Z}$ and $\mathrm{S}$ correspond to $\mathrm{W}, \mathrm{V}$ and $\Sigma$ in (2), respectively. Now, the eigenvalue decomposition of $\widehat{A}_{d}$

$$
\widehat{\mathrm{A}}_{d} \mathrm{Q}=\mathrm{Q} \Lambda \text {, }
$$

can be readily performed. The eigenvalues $\mu_{i}$ are directly obtained from $\Lambda$ in (14) and its eigenvectors can be computed from (see [9] in detail)

$$
\mathrm{P}=\mathrm{C}_{j, 2: m} \mathrm{ZS}^{-1} \mathrm{Q}
$$

Consequently, the vector potential $\boldsymbol{a}\left(i_{\mathrm{d}}, i_{\mathrm{q}}, \theta\right)$ can be expressed as

$$
\boldsymbol{a}\left(i_{\mathrm{d}}, i_{\mathrm{q}}, \theta\right) \approx \widehat{\mathrm{W}} \sum_{j=1}^{4} \mathrm{P}_{j} e^{\frac{\ln \Lambda_{d}}{\Delta \theta} \theta} \boldsymbol{b}_{j} N_{j}\left(i_{\mathrm{d}}, i_{\mathrm{q}}\right)
$$

In the preprocessing of the dynamic simulation, $\widehat{W}, \mathrm{P}_{j}, \Lambda_{d}$ and $\boldsymbol{b}_{j}$ are computed by FEM, singular value decomposition, DMD and stored, as shown in Fig. 1.

In the conventional fast dynamical simulation, the magnetic field has to be re-computed from the currents obtained by solving the circuit equation. In contrast, in the proposed method, 
the field-related quantities can be directly computed from the coefficients $\boldsymbol{c}\left(i_{d}, i_{q}, \theta\right)$ for any input current and mechanical angle through the interpolation given by (16). For example, the Maxwell stress tensor can be directly computed from

$$
\mathrm{T}\left(i_{\mathrm{d}}, i_{\mathrm{q}}, \theta\right)=\boldsymbol{c}\left(i_{d}, i_{q}, \theta\right)^{\mathrm{t}} \widehat{\mathrm{W}}^{t} \mathrm{~N} \widehat{\mathrm{W}} \boldsymbol{c}\left(i_{d}, i_{q}, \theta\right)
$$

where $\mathrm{N}$ denotes a sparse matrix including the derivatives of the interpolation function of FEM in the real space defined by

$$
\begin{array}{r}
\mathbf{N}=\frac{1}{2 \mu}\left[\begin{array}{cc}
\boldsymbol{N}_{y}^{t} \boldsymbol{N}_{y}-\boldsymbol{N}_{x}^{t} \boldsymbol{N}_{x} & -2 \boldsymbol{N}_{x}^{t} \boldsymbol{N}_{y} \\
-2 \boldsymbol{N}_{x}^{t} \boldsymbol{N}_{y} & \boldsymbol{N}_{x}^{t} \boldsymbol{N}_{x}-\boldsymbol{N}_{y}^{t} \boldsymbol{N}_{y}
\end{array}\right], \\
\boldsymbol{N}_{u}=\left[\begin{array}{llll}
\frac{\partial N_{1}}{\partial u} & \frac{\partial N_{2}}{\partial u} & \cdots & \frac{\partial N_{n}}{\partial u}
\end{array}\right]^{t}, \quad u=\{x, y\} .
\end{array}
$$

\section{FIG. 1 HERE}

\section{NUMERICAL RESULTS}

We apply the present method to the analysis of an electric motor shown in Fig. 2 which has 4 poles and 24 slots (D model of IEEJ) to verify its effectiveness. The MOR-related parameters are summarized in TABLE I, where $s_{0}$ is determined so that the cumulative contribution rate in singular value decomposition exceeds 0.999 . The current effective value and the current phase angle are set to 4.0 Arms and $30 \mathrm{deg}$, respectively. The interval $\Delta \theta$ of mechanical angle for computation is 0.5 degrees. Since this motor has 24 slots and 4 poles, it generates torque at an electrical angle of 1/6 cycle. Thus, the torque is evaluated in the range $15 \leq \theta \leq 45$ degrees.

The torque waveforms obtained by the conventional FEM and proposed method are shown in Fig. 3. It is found that both torque waveforms are in good agreement. It is remarkable that the interpolation of the proposed method works fairly well.

In Fig. 4, the average torques computed by the conventional FEM and proposed method are plotted against the current phase angle. It can be seen that both torques are consistent over all the conditions. In the design of electric motors, it is important to know the condition for the maximum average torque. The proposed method is also useful for this purpose.

Fig. 5 depicts the spatial distribution of the radial electromagnetic force at $\theta=15.0,45.0$ and 75.0 degrees. We find again the good correspondence between the results obtained by the conventional FEM and the proposed method. Table II summarizes the computing times for one electrical angle cycle including and excluding pre-processing of the input files. The proposed method is more than ten times faster than the conventional FEM. In the dynamic computation, the proposed method per one operation takes just about $20 \mathrm{~ms}$ to restore the magnetic field when using AMD Ryzen Threadripper2990WX@3.00Ghz memory 64GB.

Finally, we discuss the effects of the number of basis vectors $s_{0}$ in POD and snapshot interval $\Delta \theta$ on the result. Fig. 6 shows the effect of $\Delta \theta$ on the torque waveform. For each value of $\Delta \theta$, $s_{0}$ is determined so that the cumulative contribution rate exceeds in singular value decomposition 0.999 . It can be seen from Fig. 6 that it is sufficient to have an accurate waveform if $\Delta \theta \leq 1^{\circ}$. This condition would depend on the contribution from the higher harmonics to the waveform. Fig. 7 shows the comparison of torque waveforms for different values of $s_{0}$. It is remarkable that the wavefrom is still well restrored even if $s_{0}$ is reduced to 100 . This means that it suffices to store 100 basis vectors and corresponding weight coefficients to correctly restore the torque waveform.

\section{FIG. 2-7 HERE TABLE 1,2 HERE}

\section{CONCLUSION}

In this paper, a simplified POD-based MOR has been proposed. In this method, the field is restored via interpolation of the coefficients for the basis vectors without solving the reduced equations. The interpolation is performed on the space spanned by the input currents. The change of the coefficient along the mechanical angle is expressed using the interpolation with the DMD. The field can be restored in the dynamic simulation within around $20 \mathrm{~ms}$. The proposed method has been shown to yield the correct torque waveform when the sampling interval is set to adequate value, 1 degree for the numerical example. The number of the basis vectors for POD can be reduced to 100 to restore the torque waveform.

In future, we will apply the proposed method to the vibration analysis of electric motors. We will also study the automatic determination of the intervals in the grid on the $i_{d}-i_{q}$ plane.

\section{REFERENCES}

[1] O.A. Mohammed, S. Liu, Z. Liu, "Physical Modeling of PM Synchronous Motors for Integrated Coupling with Machine Drives", IEEE Trans. Magn. vol.41, no.5, 1628 - 1631, 2005.

[2] H. Kaimori, K. Akatsu, "Behavior Modeling of Permanent Magnet Synchronous Motors Using Flux Linkages for Coupling with Circuit Simulation," IEEJ J. Industry Applications, vol.7, no.1, 56-63, 2018

[3] T. Henneron and S. Clenet, "Model order reduction applied to the numerical study of electrical motor based POD method taking into account rotation movement," Int. J. Num. Model, Vol.27, No.3, pp.485494, 2014.

[4] T. Shimotani, Y. Sato, T. Sato, H. Igarashi, "Fast Finite-Element Analysis of Motors Using Block Model Order Reduction," IEEE Trans. Magn., vol. 52, no. 3, art no.7207004, 2016.

[5] Y. Paquay, O. Bruls, and C. Geuzaine, "Nonlinear Interpolation on Manifold of Reduced-Order Models in Magnetodynamic Problems," IEEE Trans. Magn., vol.52, no.3, art no.7204804, 2016.

[6] M. Farzamfar, F. Martin, A. Belahcen, L. Monitier, and T. Henneron, "Orthogonal Interpolation Method for Order Reduction of a Synchronous Machine Model," IEEE Trans. Magn., vol.54, no.2, art no 8100506, 2018.

[7] K. Okamoto, H. Sakamoto, H. Igarashi, "Dynamic Simulation of Electric Motor Using Simplified Model Order Reduction Based on POD", submitted for presentation at COMPUMAG2019

[8] P.J. Schmid, "Dynamic mode decomposition of numerical and experimental data", J. Fluid Mechanics, Vol. 656, 2010, pp. 5-28

[9] J. H. Tu, C. W. Rowley, D. M. Luchtenburg, S. L. Brunton, and J. N. Kutz. "On dynamic mode decomposition: Theory and applications," Journal of Computational Dynamics, 1(2):391421, 2014

[10] S. Paul, J. Chang, "Fast Numerical Analysis of Electric Motor Using Nonlinear Model Order Reduction," IEEE Trans. Magn., vol. 54, no. 3, art no.8101404, 2018. 
TABLE I

PARAMETERS OF MOR

\begin{tabular}{cc}
\hline $\mathrm{n}:$ number of FEM node & 16557 \\
$\mathrm{sm}:$ number of snapshot & 11584 \\
$\mathrm{~s}_{0}:$ low rank approximation number & 640 \\
range of mechanical angle[degree] & $15 \sim 195$ \\
snapshot interval $\Delta \theta$ [degree] & 1 \\
range of $i_{\mathrm{d}}[\mathrm{A}]$ & $0 \sim-7$ \\
range of $i_{\mathrm{q}}[\mathrm{A}]$ & $0 \sim 7$ \\
snapshot interval id,iq[A] & 1 \\
\hline \hline
\end{tabular}

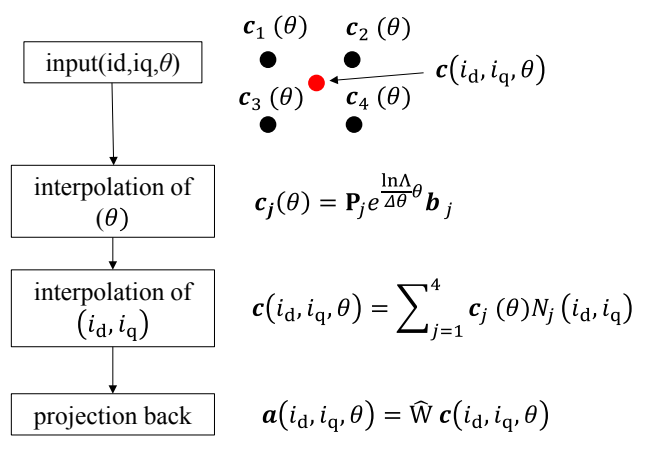

Fig.1. Procedure of the proposed method

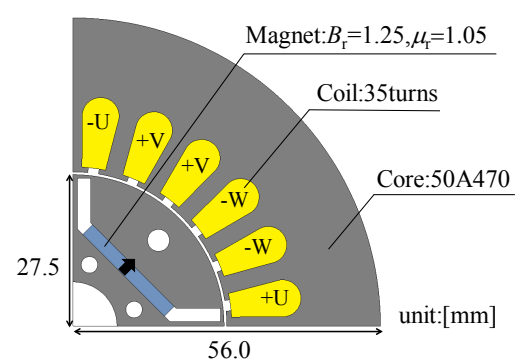

Fig.2. electric motor for numerical example

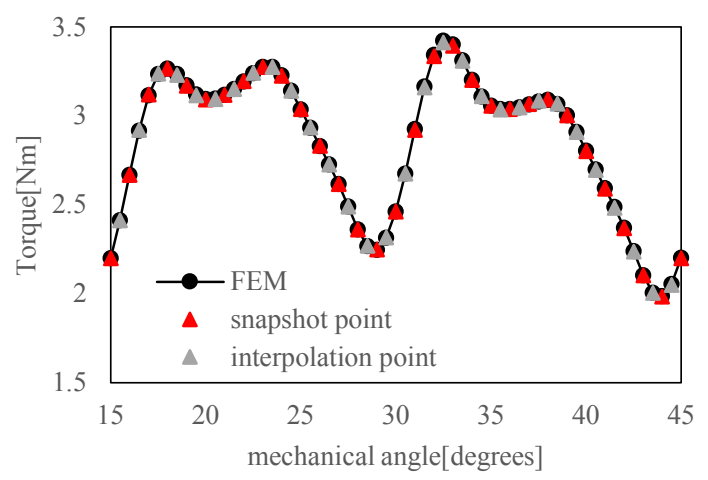

Fig.3. Torque curve (current effective value 4.0Arms, current phase angle 30deg)
TABLE II

COMPUTING TIMES.

\begin{tabular}{ccc}
\hline \hline Computation Method & Time[s] & speed up rate \\
\hline FEM & 861 & 1 \\
Proposed Method & $8.0(3.6)$ & $107.6(239.2)$ \\
\hline \hline Parenthesized times do not include pre-processing.
\end{tabular}

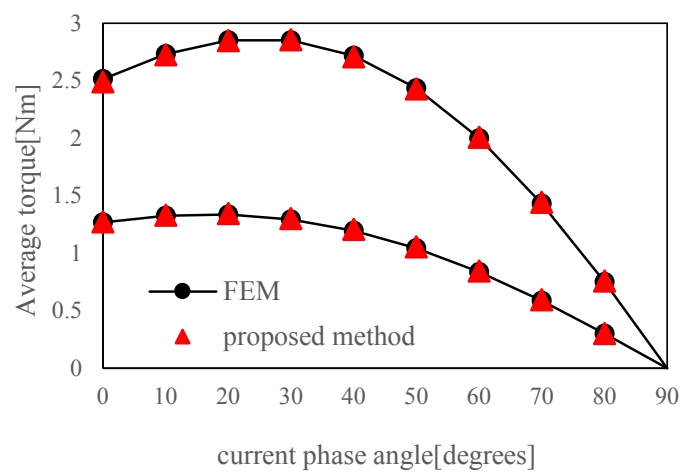

Fig.4. Dependence of average torque on current phase angle

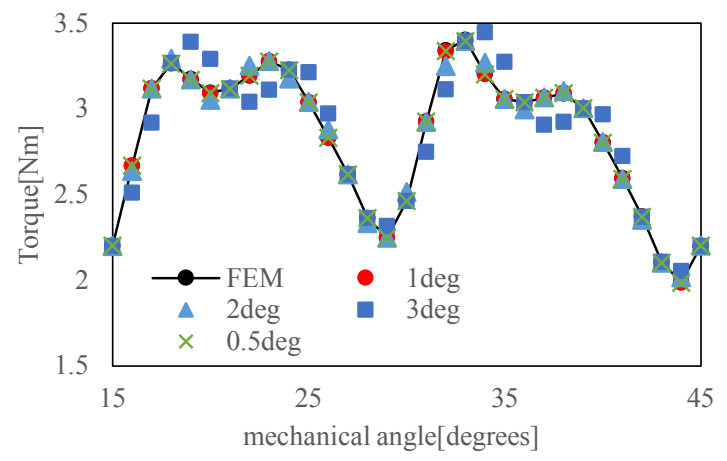

Fig.6. Effect of snapshot interval $\Delta \theta$

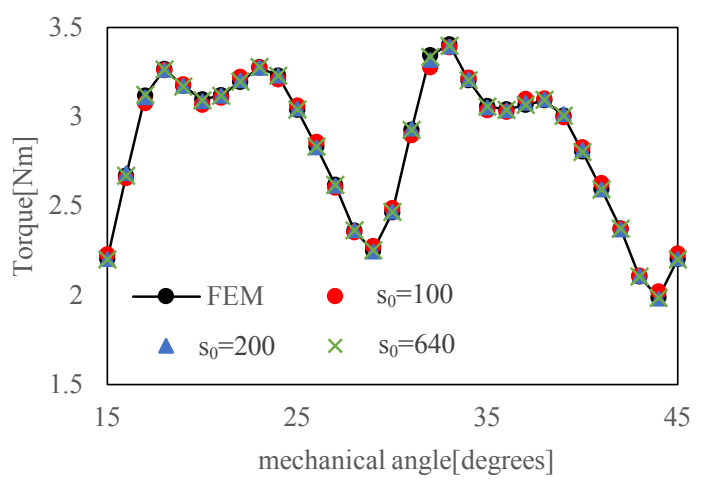

Fig.7. Effect of number of basis vectors $s_{0}$ in POD

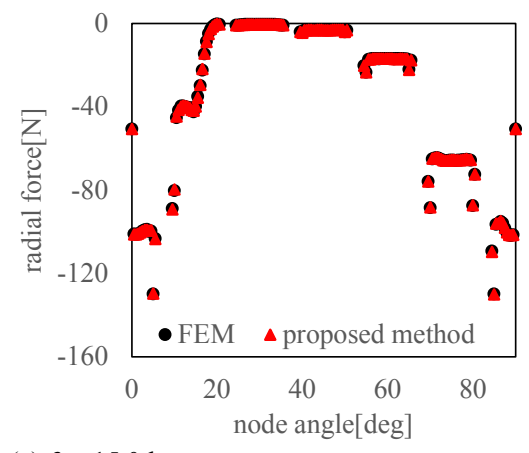

(a) $\theta=15.0 \mathrm{deg}$

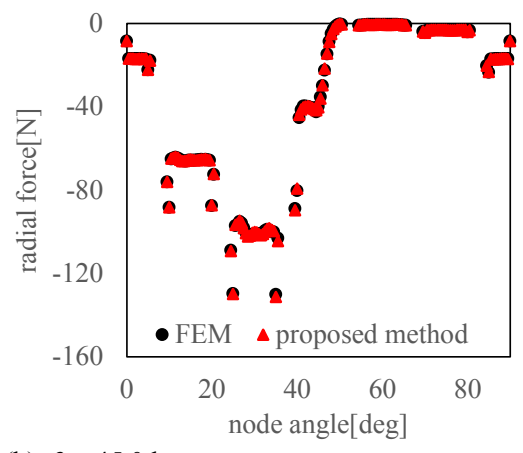

(b) $\theta=45.0 \mathrm{deg}$

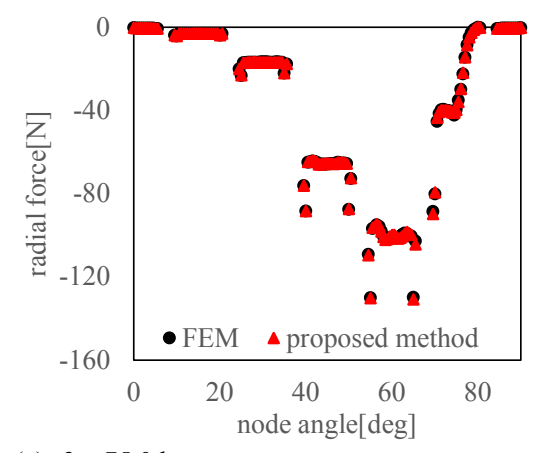

(c) $\theta=75.0 \mathrm{deg}$

Fig.5. Radial electromagnetic forces acting on the stator teeth computed by FEM and the proposed method for different mechanical angles $\theta$. 